\title{
Sarcoidosis presenting with intermediate uveitis and subcutaneous nodules
}

\author{
Dani Cadieux MD, Rishi Gupta MD
}

Cite as: CMAJ 2019 September 9;191:E1006. doi: 10.1503/cmaj.190152

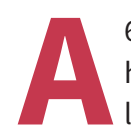

61-year-old woman presented with a 3-month history of floaters and decreased vision in her left eye. Her visual acuity was $6 / 7.5$ and 6/18 in the right eye and left eye, respectively. Dilated examination of the left eye showed granulomatous inflammation involving the vitreous and retina (Figure 1) consistent with intermediate uveitis. In follow-up, the patient reported constitutional symptoms of weight loss, night sweats, malaise and fatigue. She also noted several subcutaneous nodules on the extensor surface of her right forearm that had increased in size since the onset of her visual symptoms (Appendix 1, available at www.cmaj. ca/lookup/suppl/doi:10.1503/cmaj.190152/-/DC1). Laboratory investigations showed an elevated angiotensinconverting-enzyme (ACE) level of 93 (normal range 8-52) U/L. Skin biopsy of a lesion from the patient's proximal forearm showed subcutaneous sarcoidal-type granulomatous inflammation consistent with sarcoidosis. Although the initial chest radiograph was normal, follow-up computed tomography (CT) showed hilar and mediastinal adenopathy. We diagnosed sarcoidosis and started the patient on oral prednisone treatment. The subcutaneous nodules resolved; however, ongoing treatment with methotrexate was required owing to the return of her visual symptoms during steroid tapering.

When the vitreous is the major site of inflammation in the eye, the condition is classified as intermediate uveitis. ${ }^{1}$ The differential diagnosis of intermediate uveitis includes infection, inflammatory disease, multiple sclerosis, lymphoma and sarcoidosis. ${ }^{2}$

Sarcoidosis is a systemic inflammatory disease of unknown etiology characterized by noncaseating granulomas. ${ }^{3}$ Intraocular inflammation is present in $30 \%-60 \%$ of patients. ${ }^{2}$ Sarcoidosis with subcutaneous nodules, also known as Darier-Roussy sarcoidosis, accounts for $1.4 \%-16 \%$ of cases of cutaneous sarcoidosis. ${ }^{4}$

The presentation of uveitis with subcutaneous nodules is uncommon. ${ }^{4}$ According to the International Workshop on Ocular Sarcoidosis, initial investigations for a patient with suspected ocular sarcoid- osis include a tuberculin skin test, determination of the ACE or serum lysozyme level, liver enzyme tests, chest radiography and, in patients with negative findings on chest radiography, chest $\mathrm{CT} .{ }^{2}$ Given the multisystem involvement of sarcoidosis, when visual symptoms are present in a patient with suspected sarcoidosis, early referral to an ophthalmologist is suggested to rule out ocular involvement.

\section{References}

1. Jabs DA, Nussenblatt RB, Rosenbaum JT; Standardization of Uveitis Nomenclature (SUN) Working Group. Standardization of uveitis nomenclature for reporting clinical data. Results of the First International Workshop. Am J Ophthalmol 2005;140:509-16.

2. Herbort CP, Rao NA, Mochizuki M; members of Scientific Committee of First International Workshop on Ocular Sarcoidosis (IWOS). International criteria for the diagnosis of ocular sarcoidosis: results of the first International Workshop On Ocular Sarcoidosis (IWOS). Ocul Immunol Inflamm 2009;17:160-9.

3. Soto-Gomez N, Peters JI, Nambiar AM. Diagnosis and management of sarcoidosis. Am Fam Physician 2016;93:840-8.

4. Ando M, Miyazaki E, Hatano Y, et al. Subcutaneous sarcoidosis: a clinical analysis of nine patients. Clin Rheumatol 2016;35:2277-81.

\section{Competing interests: None declared.}

This article has been peer reviewed.

The authors have obtained patient consent.
Affiliation: Department of Ophthalmology and Visual Sciences (Cadieux, Gupta), Dalhousie University, Halifax, NS

Correspondence to: Dani Cadieux, danicadieux@dal.ca 